\title{
Association between habitual school travel and muscular fitness in youth
}

ARTICLE in PREVENTIVE MEDICINE · JULY 2014

Impact Factor: 2.93 · DOI: 10.1016/j.ypmed.2014.07.036 · Source: PubMed

DOWNLOADS

3

6 AUTHORS, INCLUDING:

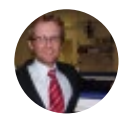

Matthew J D Taylor

University of Essex

36 PUBLICATIONS 213 CITATIONS

SEE PROFILE

Dominic Micklewright

University of Essex

36 PUBLICATIONS 143 CITATIONS

SEE PROFILE
VIEWS

42
Christine Voss

University of British Columbia - Vancouver

46 PUBLICATIONS 209 CITATIONS

SEE PROFILE

Gavin R H Sandercock

University of Essex

109 PUBLICATIONS 1,077 CITATIONS

SEE PROFILE 
Elsevier Editorial System(tm) for Preventive Medicine

Manuscript Draft

Manuscript Number: PM-13-896R1

Title: Association between habitual school travel and muscular fitness in youth.

Article Type: Original Research Paper

Keywords: HANDGRIP; PEAK POWER; CYCLING; ACTIVE TRAVEL; PHYSICAL ACTIVITY, ADOLESCENTS; CHILDREN

Corresponding Author: Dr Gavin RH Sandercock, Ph.D

Corresponding Author's Institution: University of Essex

First Author: Daniel D Cohen, PhD.

Order of Authors: Daniel D Cohen, PhD.; Ayodele A Ogunleye, PhD.; Dominick Micklewright, PhD.;

Matthew J Taylor, PhD.; Christine Voss, PhD.; Gavin RH Sandercock, Ph.D

Abstract: Objective: To determine whether active school travel is associated with muscular fitness, which is an emerging marker of youth health. Methods: Handgrip strength, vertical jump and vertical jump peak power were measured in $n=6829$ English schoolchildren ( $53 \%$ males, age $12.9 \pm 1.2$ years) between 2007 and 2011. Participants were grouped according to self-reported habitual school travel modality. Results: Cyclists had greater handgrip strength than passive travelers. Vertical jump height was greater in walkers and cyclists compared with passive travelers. Jump peak power was also higher in walkers than in the passive travel group. Compared with passive travelers, cyclists had a higher (age, sex and BMI-adjusted) likelihood of good handgrip strength (OR 1.42, 95\%CI;1.14-1.76) and walkers were more likely to have good measures for vertical jump peak power (OR 1.14, 95\%CI;1.00-1.29). Cyclists' likelihood of having good handgrip strength remained significantly higher when adjusted for physical activity (OR 1.29, 95\%CI;1.08-1.46). Conclusion: Muscular fitness differs according to school travel habits. Cycling is independently associated with better handgrip strength perhaps due to the physical demands of the activity. Better muscular fitness may provide another health-related reason to encourage active school travel. 
Prev med cover 2

Dear Reviewers,

Thank you for taking the time to review our manuscript and provide us with feedback. It is rare to get such detailed and considered feedback and we very much appreciate the amount of time and effort you have put into reviewing our work. Thank you.

We very much value your comments, which have been extremely useful in helping us to revise and improve the manuscript. Please find below a point-by-point explanation of how we have addressed each of your comments, which we hope you will find satisfactory. Once again, many thanks for your hard work.

We would like to point out that due to inevitable differences in feedback between (three) reviews and the word-count restrictions, we have had to respond to some comments here alone, as opposed to doing so in the manuscript itself.

Finally, these comments and responses run to 15 pages, the manuscript is so different to the original submission we could not see the value of giving page and line references. The reviewer's original comments still have these of course but they now bear little resemblance to the placement of responses in the manuscript.

The Authors. 
Responses to Comments from Editorial Office:

1) Our publisher's optional "Your paper, your way" submission feature simplifies the work of preparing and uploading manuscripts for publication in Preventive Medicine. This allows authors to submit to our journal a paper that they prepared initially using a different format than the one we require for Preventive Medicine. Authors appreciate this convenience. However, once the manuscript passes an initial review round and is returned to authors we request that the revision be formatted as follows: (i) combine tables and figures at the end of the document following the references; (ii) format references in the text and as a list at the end strictly as per the journal style; (iii) structure the abstract and main narrative using headings as per our journal style; (iv) include word counts for abstract and text (exclusive of in-line references) from beginning of introduction to end of conclusions. Consult our instructions to authors and a recent issue of Preventive Medicine for guidance on how to comply with our formatting and stylistic requirements.

Response: Thank you, we have now re-formatted the manuscript as you have requested.

2) We are concerned that your manuscript is already lengthy enough at nearly 3500 words. Although you will have to address the reviewers' concerns in the narrative please keep the entire text to 3500 words or less (from beginning of Introduction to end of Conclusions, excluding in-text citations). Include word counts for the abstract and for the main text on the cover page.

Response: Thanks you, in revising the manuscript and addressing the reviewers concerns we have managed to constrain the word count to 2895. As requested we have added an abstract and main text word count to the cover page.

3) Remove the asterisk-flagged indications of significance from table 3 . The confidence intervals already convey the information on significance.

Response: Table 3 has now been altered as suggested.

4) Report all ORs and 95\% confidence bounds with two decimal places only.

Response: All OR's and Cl's have been reduced to 2 decimal places as suggested throughout the manuscript text and in the Tables.

Response to Reviewer 1 Comments:

Reviewer \#1: This paper describes the association between self-reported travel mode to school and measures of muscular fitness in a large sample of English young people. The authors provide a reasonable argument as to why studying this association is important, present and analyse the data appropriately and put the results into the context of existing literature. The paper is reasonably wellwritten, although contains typographical errors throughout and the discussion appears overly long. I have few comments for the authors' consideration:

Response: Many thanks, we are pleased you like the manuscript and are grateful for your comments that we have addressed as highlighted below. As you have suggested we have corrected the typographical errors and reduced the length of the discussion. 
Major comments:

1. The discussion section is currently very long and not very focused. Please reduce this section. Moreover, the section of Potential Health Benefits is oddly placed in the discussion and I propose to merge it into the introduction in a shortened version.

Response: On reflection we agree that a shortened version of the potential health benefits should be moved from the discussion to the introduction as it strengthens the rationale for the study. We have deleted the potential health benefits subsection from the discussion and added a condensed version of the following text to the introduction:

'In youth handgrip's relationship with metabolic health appears particularly robust when it is expressed as a categorical variable with lower handgrip defined as a score in the lower tertile or quartile of age and gender specific performance adjusted for body mass (Artero, et al., 2011, Ruiz, et al., 2009, Cohen et al., 2014). Clinically relevant or tentative cut- points "healthy muscular fitness zones" have recently been proposed (Ortega, et al., 2012) as handgrip strength during adolescence is associated not only with good current metabolic health (Artero, et al., 2011) but also future risk of CVD and total mortality (Ortega, et al., 2012). Handgrip strength may act as a proxy of whole-body muscularity (Sherriff, et al., 2009) and may be a useful addition to estimates of adiposity such as BMI. There is some evidence that, when used in isolation, BMI values are insensitive to changes in body composition and unable to detect secular increases in children's adiposity (Moliner-Urdiales, et al., 2010). We have previously reported that BMI remained stable over the 10-year period among children despite large, secular declines in muscular endurance (-30\% for sit-ups performance) and strength (-8\% handgrip) (Cohen, et al., 2011). A systematical review reported global declines in lower body power (Tomkinson, et al., 2003) that are worrying given the emerging importance of muscular fitness as markers of current and future health.'

Minor comments:

2. There are multiple writing errors in the current paper (e.g. additional/forgotten words, spelling mistakes etc). Please correct these throughout.

Response: The manuscript has been thoroughly proof-read and the typographical errors have now been corrected.

3. P6, please provide a reference for the UK reference data for handgrip strength.

Response: This is now included in page six (Cohen, DD, Voss, C, Taylor, MJD, Stasinopoulos, DM, Delextrat, A, Sandercock, GRH, 2010. Handgrip strength in English schoolchildren. Acta Paediatrica. 99, 1065-1072). Thank you.

4. P7: distance to school was dichotomised at $2.4 \mathrm{~km}$. Previous evidence indicates that primary schoolchildren travel actively over much shorter distances to school than secondary schoolchildren. The study appears to include both primary and secondary schoolchildren. Please provide a rationale for combining the data or consider applying age-specific (school-specific) splits.

Response: Thank you this is a good point and we agree that in most cases the distance travelled by secondary school children tends to be more varied compared to primary school children who tend to travel less distance. However, we specifically chose a simple $2.4 \mathrm{~km}$ split because we wanted to 'directly' differentiate on the basis of 'real' distance rather than using a primary/secondary split with 'indirect/assumed' differences in travel distance. Using a primary/secondary school introduces different problems such as the inclusion of primary school children who travel long distances and, more problematic, numerous secondary school 
schildren who travel less than $2.4 \mathrm{~km}$. For this reason we would prefer to retain the $2.4 \mathrm{~km}$ split method but, as you have suggested, have now incorporated the following short rationale into the manuscript text:

'We dichotomized journey length as 'long' or 'short' based on median $(2.4 \mathrm{~km}) \mathrm{split}$, in order to differentiate on the basis of measured distance rather travelling distance differences sometimes inferred from other methods such as primary-secondary school or age-related splits.'

5. Please include a section heading 'statistical analyses' in the methods section.

Response: This has now been added.

6. Results: please avoid repeating all results from the tables in the text.

Response: This is now done. We agree with the reviewer who we assume is referring to table 2. We have now only included results in the table that we think may be of particular interest to readers.

7. Table 2/3: please present values with 2 decimals (as in Table 1). Also the title of Table 2 appears to be wrong - the data presented is not difference in means, but the means within groups.

Response: All values have been changed to two decimal places in Tables 2 and 3 . The title of Table 2 has also been changed to, 'Table 2. Means z-score $(95 \% \mathrm{Cl})$ for three indices of muscular fitness for walking, cycling or using passive school transport.'

8. P10: please rephrase the interpretation of an OR ('Cyclists had $42 \%$ higher odds...').

Response: We have rephrased this sentence as recommended:

'Cyclists were more likely (OR=1.42, $95 \% \mathrm{Cl} ; 1.14$ to 1.76$)$ to have good handgrip compared with the passive travel group.'

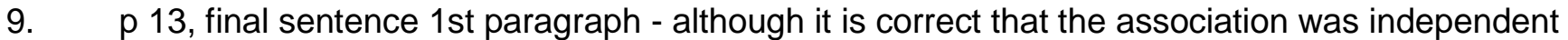
of physical activity, adjustment for physical activity did attenuate the results. The authors may want to include this in their considerations.

Response: We have now altered this sentence to recognize this point:

'...found the higher likelihood of good peak power was independent of age, sex, and BMI but that this effect was attenuated when we controlled for boys' physical activity (Model 2).'

Response to Reviewer 2 Comments:

Title

Association between habitual school travel and muscular fitness in youth

General comments: 
This study describes impact of commuting to school on two measures of muscle fitness in a large cohort of 6829 schoolchildren. I think this study identifies an interesting and relevant area of children's health and the question raised is clear and of importance. Findings supporting the promotion of active commuting are of great value in the public health perspective. Even though, I have some major concerns that I recommend the authors to consider before publication.

I will first give some overall comments and these will be followed by specific comments on minor issues.

Response: Many thanks, we appreciate your efforts in reviewing our work and are grateful for your comments that we have attempted to address and believe significantly improve the manuscript. We hope that you find the changes we have made, outlined below, satisfactory.

\#1 I do not quite comprehend with the word lower body power and moreover the authors seems to use several different terms when referring to lower body power (i.e.; jumping, lower body power, lower body-power, jump height and peak power). Please be consistent.

Response: See response under comment 2

\#2 Moreover it is difficult to understand what the authors refer to as lover body power, jump height or peak power or a combined score? In the introduction the authors state that the aim of the study was to determine if active school travel was associated with two measures of muscle fitness (handgrip strength and lower-body power) whereas in the results the authors present three different muscle fitness measures (handgrip, jump height and peak power). Please clarify.

Response \#1 \& \#2: Thank you this is a very good point and we have now removed all reference to lower body power and added the following clarification to page 4 to define key terms:

'Collective muscular fitness can be assessed using various performance tests of strength such as handgrip, explosive lower-limb power such as jumps, and muscular endurance such as situps (Artero, et al., 2011).

In the methods section under the subheading muscular fitness measures we have also described exactly how handgrip strength was measured as well as how vertical jump was measured in units of $\mathrm{cm}$ as well as converted to jumping peak power $\left(\mathrm{W} \cdot \mathrm{kg}^{-1}\right)$ based on the Sayers equation.

As recommended, we have also ensured that terms are used concisely and with precision throughout the manuscript, which we have now confined to the following terms only, as defined above: Muscular fitness, muscular endurance, handgrip strength, vertical jump and vertical jump peak power.

\#3 At the end of the first paragraph in the introduction the authors state that muscular fitness, like cardiorespiratory fitness appears to play a cardioprotective role in overweight and obese individuals. Could have been interesting to have the author's opinion on whether these associations are exclusively for overweight and obese individuals?

Response: Based on the mechanism by which cardiorespiratory fitness may be cardioprotective, the answer to your question is No. Studies and interventions have mainly focused on overweight and obese individuals, but this benefit are not exclusive to this groups. We however cannot say much for the underweights.

Research shows that cardio- respiratory fitness (fitness) attenuates the negative health consequences of high adiposity (fatness) in adults and the actual mechanisms in with cardiorespiratory fitness is linked with better cardiovascular profile (cardioprotection in this 
case) are not fully understood. However, The benefits that cardiorespiratory fitness has on the vasculature are believed to be mediated by endothelial progenitor cells, which support vascular repair (Steiner S. et al. (2005) Endurance training increases the number of endothelial progenitor cells in patients with cardiovascular risk and coronary artery disease. Atherosclerosis 181, 305-310 and Seals DR, Desouza CA, Donato AJ et al. (2008) Habitual exercise and arterial aging. J Appl Physiol 105, 1323-1332). ). A healthy blood vessel requires an intact endothelium and a degree of elasticity. High fitness is associated with lower arterial stiffness and greater arterial compliance and may decrease total resistance resistance (Pescatello LS. et al. (2004) American College of Sports Medicine position stand. Exercise and hypertension. Med Sci Sports Exerc 36, 533553).

WE HAVE then further shortened this to create the following summary:

We have changed this sentence to "such measures are becoming recognized as important health outcomes, particularly in overweight and obese children". This is to reflect that the cited studies which examined the interactions between muscular fitness, metabolic health and weight status in children have either shown a stronger negative association between strength and metabolic risk in the overweight/obese than in the normal weight (Artero et al, 2011 and Steene-Johannessen et al., 2009), or that the association was only observed in children in the upper tertile of \% body fat (Cohen et al., 2014).

\#4 In the statistic section the authors mention VO2peak for the first time and results and comparison of V02peak results between boys and girls are described in the first paragraph in the result section. However, this measure is not described in the methods section and data is not reported in table 1 . The question is whether the measure is relevant for the paper or should the authors focus on muscle fitness.

Response: This was an oversight. This is now done, thank you.

\#5 I have some concerns on the z-scores reported in table 1. Is it relevant display mean z-values? If the z-score from a large similar population as the reference population are computed by sex and age the mean z-values is always near zero. Moreover, I did not quite comprehend with the paragraph on page 8 is this information relevant for the purpose of the manuscript.

Response: We recognize that z-socres appear somewhat unsightly and may be difficult to interpret but we need to present means (sd) of our outcome variables to adhere to the journal policy on providing information necessary to supporting potential future meta-analyses. We have removed the point on 'means near zero and SD1 as you rightly point out, this is superflouous.

\#6 In the literature several studies compute composite scores as an estimate of overall muscular fitness (1-3) when several measures of different terms of the muscle fitness concept are available. It is my opinion that by creating sum scores different dimensions of muscle strength are taken into account. That might give a more precise description of overall muscle fitness than just single measure of either isometric strength or power. In the present study the authors have both isometric strength and explosive strength/power. Could the authors comment on why they do not use a composite score for muscle fitness instead of single measures. It could at least be interesting to have some kind of analyses on a composite score.

Response:

We agree that a composite score could be an interesting addition to the analysis. However, we believe that providing analysis of individual components is important since vertical jump and handgrip not only represent different components of muscular fitness, access to these measures also differ - clearly, in the school setting the assessment of vertical jump height may 
be done without any additional equipment, while handgrip dynamometers are less widely available. Furthermore, while there is substantial evidence showing associations between both handgrip and standing long jump and cardiometabolic health in youth, to the best of our knowledge this has not been reported for vertical jump.

We actually submitted a manuscript (on a another topic) containing a composite fitness score manuscript to this journal last year which was rejected due to a lack of scientific rationale our worry in doing so again would be how to 'weight' different scores? Would each score be weighted equally and would we include: cardiorespiratory measures, or measures of adiposity like BMI as others have?

The wording necessary to explain the rationale for any such score would use too much additional space and add to our word count.

\#7 One other concern is the age span in the study. One might assume that the travel pattern is different in different age groups. Could the authors comment on whether there are more walkers in lower age groups and more cyclers in older age groups, and finally is the pattern of passive transport different over the age span? Final question is whether these differences are taken in to account in the analyses.

Response: We can comment here, but word count does not permit us to address this valid point. Yes more of the younger children walk, and more use active travel when older. We cannot control for this confounder; the prevalence of all modes differs widely between schools which is why we used multilevel model. We now briefly address this in the limitations.

'Given the exploratory nature of our investigation we maximized statistical power by analyzing all participants using age- and sex-specific z-scores and further statistical adjustment. This analysis does not account for age-related differences in travel habits. Future research would benefit from analyses stratified by age but would require very large samples or deliberate oversampling of cyclists to increase statistical power'

\#8 Although validated I would very much appreciate the authors comment on using a 7-day recall instrument in schoolchildren, especially among the youngest group. Is it useful? And what is acceptable criterion validity?

Response: We have added the value for criterion validity in methods and a brief comment on limitations of self-report is now in the limitations

\#9 The authors need to discuss to a broader extent the association between handgrip strength and cycling. Is there any biological explanation for the association between cycling and handgrip strength. Handgrip is often used as an indirect measure of overall strength, at least for the upper body. The authors state that the relationship may be a consequence of holding handlebars and using brakes. I am not sure if I can support this explanation, as holding handlebars often will be characterized as an static exercise, however, one might assume that off-road cycling could give such an adaption to the muscles. On the other hand I doubt English adolescent do off-road cycling when commuting to school. What surprises me is that cycling was more related to handgrip than lower body power. One might assume that overall activity; walking, cycling or other activities should have an effect on musculoskeletal system in the present age group. Could the findings or lack of finding on the relationship with cycling and lover body power be due to low number of cyclist and thereby low statistical power?

Response: Indeed holding of handlebars and squeezing brakes are very similar to the muscle 
activation pattern of the handgrip test which involves isometric contraction of the forearm muscles (albeit at a submaximal intensity).

It is possible as suggested by Artero et al, that the vertical jump is test which depends to a great extent on coordination of the muscle groups involved, and as such the power development in the thigh muscles that may occur during cycling, does not transfer to the performance of the single effort maximal test. We have mentioned both of these possibilities in the text and in the limitations that that the small number of cyclists may limit statistical power to detect this relationship. We hope that this is sufficient.

\#8 From the last paragraph on page 13 and to the end of page 14 the authors comment on several important issues with respect to potential health benefit of muscle fitness. However, I question whether this is beyond the scope of the manuscript? Some of these aspects are already mentioned in the introduction.

Response: Removed as per reviewer 1 comment 1.

Specific comments:

\#1 Page 5 last paragraph -please write body mass index when it is the first time the term is being used

Response: Thank you.

\#2 Page 6 second paragraph - please insert reference for the UK reference data used for expressing z-score

Response: This is now included.

\#3 Handgrip strength converted to relative strength (W.kg-1) according to the methods section, however, only absolute values are reported in table 1 . Why not? This might be the most correct way to express handgrip strength - especially when comparing boys and girls?

Response:

Generally, raw handgrip is reported in descriptive tables (see refs below) while HG/kg or Zhandgrip/kg to account both for body size and gender. We are not comparing boys and girls but are happy to add HG/kg to the table if requested.

(1)Ruiz JR, Ortega FB, Warnberg J, Moreno LA, Carrero JJ, Gonzalez-Gross M, et al. Inflammatory Proteins and Muscle Strength in Adolescents: The AVENA Study. Archives of Pediatrics Adolescent Medicine. 2008;162(5):462-8.

(2)Garcia-Artero E, Ortega FB, Ruiz JR, Mesa JL, Delgado M, Gonzalez-Gross M, GarciaFuentes M, Vicente-Rodriguez G, Gutierrez A and Castillo MJ. [Lipid and metabolic profiles in adolescents are affected more by physical fitness than physical activity (AVENA study)]. Rev Esp Cardiol 60: 581-588, 2007.

(3)Steene-Johannessen J, Anderssen SA, Kolle E, Andersen LB. Low muscle fitness is associated with metabolic risk in youth. Med Sci Sports Exerc. 2009;41(7):1361-7.

Response to Reviewer 3 Comments:

Reviewer \#3: The manuscript investigates the association between travel mode to school and 
muscular fitness. The study, which is based on a large sample $(n=6,989)$ drawn from The East of England Healthy Hearts investigates an important topic. The introduction is well organized and logically put into perspective what follows in the manuscript. The methods section should be more specific and more clear in a number of aspects. Likewise should the results section be thoroughly revised e.g. it seems that some of the provided estimates are incompatible with the accompanying confidence. The discussion could generally be condensed and missing and erroneous references should be taken care of.

Response: Thank you for your comments which we are grateful for and hope that the responses we have made, as outlined below, meet with your satisfaction. We believe that the changes you have suggested have helped us to significantly improve the manuscript so thank you.

Specific comments: $(\mathrm{P}=$ page, $\mathrm{L}=$ line $)$.

1) P0.L8: Abstract: should the $\mathrm{Cl}$ interval be [1.00-1.29] ?

Response: Thank you, well-spotted. This has now been changed as suggested from 1.00-1.28 to 1.00-1.29.

2) P1,L44: Note that if you are well above the current word limit. This study does not involve comparative interventions thus the manuscript should not exceed 2500 words. Only if the rules regarding the word limit are going to be relaxed a major revision of the manuscript could be avoided.

Response: We agree with you. This has been exceeded largely because we have tried to accommodate all reviewer's comment and included details that we feel reader will be interested in seeing.

3) P3.L9: General comment. Would it be possible to use the same terminology (lower body power or peak power or relative peak power) throughout the entire manuscript?

Response: Thank you, this was also commented on by reviewer two. As recommended, we have now ensured that terms are used concisely and with precision throughout the manuscript, which we have now confined to the following terms only, as defined above: Muscular fitness, muscular endurance, handgrip strength, vertical jump and vertical jump (VJ) peak power.

4) p4, I.9-11: please rephrase so handgrip strength could not be interpreted as a measure of power

Response: The sentence has now been rephrased as suggested:

'Recently, measures of strength such as isometric handgrip strength, and power such as jump performance, have been identified as powerful indicators of children's health status independent of cardiorespiratory fitness.'

5) P4. L.26 would e.g. through or by means of - be more suitable than "on"?

6) p4,Line 27: It appears at the moment that both "power" and "strength" is related to handgrip.

7) p4,line:27-31: I suggest that you add. e.g. before "(jumping") and e.g. before "(sit-ups") so one is not mislead to think that muscular fitness solely is related to these specifically

Response to 5,6 \& 7: Thank you we have decided to rephrase this whole section for clarity as 
follows:

'Collective muscular fitness can be assessed using various performance tests of strength such as handgrip, explosive lower-limb power such as jumps, and muscular endurance such as situps (Artero, et al., 2011).'

8) P4.L:32: Hunt et al. 2011 does not establish any independent influence of fitness on systolic blood pressure and reports rather small beneficial influence on diastolic BP. I suggest using another reference - there are far more convincing studies available on this matter.

Response: Thank you we have now included other references.

9) P4.L34: It appears that muscular fitness does only play a cardioprotective role in overweight and obese. Is the protective role meant as a general phenenomen also relating to normal weight children? If so rephrasing should be considered as well as including other perhaps more suitable references than Artero $2010+2011$ in L.39 should be considered.

Response: Yes. Studies and interventions have mainly focused on overweight and obese individuals, but this benefit are not exclusive to this groups. We however cannot say much for the underweights.

Research shows that cardio- respiratory fitness (fitness) attenuates the negative health consequences of high adiposity (fatness) in adults and the actual mechanisms in with cardiorespiratory fitness is linked with better cardiovascular profile (cardioprotection in this case) are not fully understood. However, The benefits that cardiorespiratory fitness has on the vasculature are believed to be mediated by endothelial progenitor cells, which support vascular repair (Steiner S. et al. (2005) Endurance training increases the number of endothelial progenitor cells in patients with cardiovascular risk and coronary artery disease. Atherosclerosis 181, 305-310 and Seals DR, Desouza CA, Donato AJ et al. (2008) Habitual exercise and arterial aging. J Appl Physiol 105, 1323-1332). ). A healthy blood vessel requires an intact endothelium and a degree of elasticity. High fitness is associated with lower arterial stiffness and greater arterial compliance and may decrease total resistance resistance (Pescatello LS. et al. (2004) American College of Sports Medicine position stand. Exercise and hypertension. Med Sci Sports Exerc 36, 533553).

DC : As above Suggest instead the following:

We have changed this sentence to "such measures are becoming recognized as important health outcomes, particularly in overweight and obese children". This is to reflect that the cited studies which examined the interactions between muscular fitness, metabolic health and weight status in children have either shown a stronger negative association between strength and metabolic risk in the overweight/obese than in the normal weight (Artero et al, 2011 and Steene-Johannessen et al., 2009), or that the association was only observed in children in the upper tertile of \% body fat (Cohen et al., 2014).

10) P5.L7: There has in fact also been a previous Norwegian study investigating the association between muscular fitness and transport mode to school. Cf. International Journal of Behavioral Nutrition and Physical Activity 2013, 10:91

Response: Thank you for spotting this recent article. We have now accommodate this.

11) P5.L.27:Generally check the manuscript style. This primary title is for example not different from the secondary title "Anthropometry"

Response: There are no strict formatting requirements that we can find regarding this, but we 
see what you mean. This is now adjusted.

12) P5.L31:The methods in Voss 2010 solely relates to the sampling strategy in 2007 and 2008. Is this study based on the same 23 schools included in Voss 2010 . This could be made more clear.

Response: You are right, the sampling strategy was in 2007, but the data collection was ongoing during the summer month of subsequent years.

13) P4.L37: Please report how many subjects that were excluded due to missing data. Also you should report the participation rate so the risk of selection bias can be evaluated.

Response:

14) P.5.L44: please add specifically which institution and add the provided registration number from the ethical committee?

Done..

15) p6,I12:which specific equipment. It should be possible to reproduce the experiments

Response: This is now included.

16) P6.L17:which specific equipment

Response: This is now included.

17) P5.L29: Please rephrase so it is clear which kind of strength (handgrip?). Furthermore, what is meant by "..strength jump height and peak power" ?

Response: Yes we have now ensure such terms are used with greater precision and consistency here and throughout the manuscript: Muscular fitness, muscular endurance, handgrip strength, vertical jump (VJ) and vertical jump (VJ) peak power

18) P5.L.31: PAQ questionnaire description seem to be misplaced in a section about muscular fitness measures

Response: We have now incorporated this into a new subsection entitled, 'Measures of Physical Activity and School Travel.'

19) P6.L22. it is stated that the performance in the countermovement jump test is expressed as jump height $(\mathrm{cm})$ and relative peak power $(\mathrm{w} / \mathrm{kg})$. Why then is crude $\mathrm{W}$ reported in table 1 ?

Response: Thank you, we have now revised Table 1 to read, 'VJ Peak Power (W.kg-1)'

It is unclear to me if the peak power $(z)$ estimates in in table $2+3$ based on crude watt or watt relative to body mass? (This relates also to comment \#3)

Response: they are based on Crude $\mathrm{W}$ as this is the metric used in the paper containing the reference data (Taylor et al. 2012)

Note: If you decide to analyse "non"relative watt - then I suggest that you include investigate if subject mass is related to the transport mode (since large subjects would produce more power). 
Response:

Since peak power is estimated using a formula which includes body mass and jump height, to then divide by body mass seems to be redundant, while jump height is itself considered a measure of power relative to body mass.

20) P6.L.34: additional information e.g. on the range (1-5?) of this scale should be added (see also comment \#36)

Response:

21) P6.L37: I suggest the degree of validity is omitted here and instead included in the discussion section.

Response: We added this statement here for the sake of brevity. We are happy to discuss this if the editors wishes.

22) P6,L39: Why have you chosen to cite one of your own previous studies applying the PAQquestionnaire instead of the original article by Kowalski et al. 2004?

Response: This sentence was meant to lay emphasis on some of the studies we have done and not on the second part (Kowalski's work). We have however accommodated this.

23) P6.L49: Would it be better to write?: "The participants who selected "other" $(n=160) \ldots$. " or that you simply delete $" n="$

Response: Thank you for this suggestion.

24) P7.L1: where are these results presented?

Did you automate the distance calculation process or did you do it manually for each of the participants? It would strengthen the paper substantially if the distance was included in the manuscript. If this is done you could supplement with dose-response analyses. Note: When these are implemented you should provide information on whether the distance was calculated as the crow flies or as the distance based on available roads. The latter would require information on the settings/constraints of the distance calculation.

Response: Distance was calculated manually, if we understood what you meant by manual. We appreciate your comment on including distance to the manuscript, however we hope to do this in a future article as another researcher within our team is interested in this.

25) P7.L12: Why did you choose to adjust your logistic regression models - and not your linear regression models - for general level of physical activity? Are you able to elucidate residual confounding in your linear reg.models?

Response: We have used logistic regression, t-test and ANCOVA mainly for our analysis. Which linear regression?

26) P7.L.14: "...differences" in ? between ?

Response: Thank you

27) P7.L.34: should "mixed effects model" be added? 
Response: Multilevel models and mixed effect models are synonymous. Please advice.

28) P7.L.41: information about SPSS in the parenthesis does not follow the standard formulation

Response: Thank you for this observation, we have now revised this.

29) P.7.L46: please be consistent and add software developer information

Response: Done.

30) P7.L51: since this is concerning a volume rate a dot above the $V$ should be added

Response: Thank you, this is now added.

31) P9.L1. regarding table 2. The title indicates that differences between mean values are presented. If this is the case it is unclear which delta values are being presented in the table.

Response: The table only shows the mean in each sub group. The post hoc analysis is what makes the difference comparison possible and the significantly different ones from the the passive travel group are indicated with an asterisk $\left(^{*}\right)$

It would increase readability if the same order as in table 3 (passive, walking, cycling) was used 3

Response: We reach this decision after deliberating this order amongst ourselves. We would prefer it as it some that the referent group 'passive' comes first. We are happy to change this order if deemed absolutely neccessary.

32) P9.L16: What were your considerations when deciding to adjust for age, sex, BMI-z? It appear likely that adjustment for BMI-z would have rather different impact on handgrip, jump height and peak power (recalling the concepts of body scaling some of the outcomes seem to be far more dependent on body mass/composition than others).

Response: Yes, absolutely. We are attempting to control for potential differences in adiposity, and hoped that by using BMI (a ratio measure) in a large population that we wouldn't impact too much on potential differences due to body size. While it's a bit of a circular argument, controlling for BMI made only minimal adjustments to any of the measures' association with travel (in any direction). We feel that, by adjusting, we can more safely suggest that differences are not due to differences in body size/adiposity.

P7.L.24:this is unclear to me. Perhaps you specifically should make clear what is meant by "post hoc tests of marginal means (Bonferroni)..."

Response: post hoc was done for comparing the groups, specifically for patterns that were not specified a priori.

33) P9.L27: "differences in grip strength in cyclists" compared to what ?

Response: Passive transport group. Thank you.

34) P9.L22-45: This section is unclear and should generally be revised. In line 29 specifically there seem to errors in both estimates and confidence intervals presented. How can the mean value be statistically higher with the provided $\mathrm{Cl}^{\prime} \mathrm{s}$ 
Response: This is a typographical error. Thank you.

35) P10.L1: In my opinion you have only made two tests of muscular fitness (though you operate with three "measures" of muscular fitness

Response: Correct - we have changed this to "performance in three indices ... " which is also consistent with table 2.

36) P10.L10.table 3: Was physical activity to school included in the PAQ-score? If so you tend to adjust for (some of the) exposure being investigated. It would strengthen the manuscript if this was made clear.

Response: Not specifically, there is no transport question in the PAQ-A or C, as reviewer 2 asked us to explain the PAQ in more detail and we have included the following sentence 'and does not include a school transport item' to help make this point.

37) P.10.L32: Did you adjust for BMI-z or BMI

Response: BMI Z-score. Thank you.

38) P10.L34: "good handgrip.."- add strength?

Response: Done

39) P.11.L.5: incorrect reference

Response: now removed.

40) P11.L12: The authors claim that there is good evidence that children walk or cycle to school perform better in cardiorespiratory fitness (CRF) tests. To my knowledge there is not strong evidence that walking to school is associated to CRF. Your own 2010 paper seem to be the only study providing such evidence. Please correct me if I am wrong?

Response: now removed in edits - We also agree and you are not wrong. There are very few articles on this subject (active transport and cardiorespiratory fitness) and studies are on going in another population by one of the authors on this. Evidence from studies on this are not too strong methodologically as they are mainly cross-sectional in nature. Future studies are warranted.

41) P11.L.24-25: What is your rationale for interpreting the previous shown associations between travel mode to school and muscular fitness as "a connection between strength and power and measures of child health"? In this connection also be aware of the redundancy with line 8-9.

Response: This sentence was misworded, now changed.

42) P11.L56: add reference to tracking to adulthood

Response: Done.

43) P12.L14:Where in Hendriksen el al. 2000 do you find this description. I could not recognise it in the article.

Response: This was actually our interpretation of Hendriksen's findings - given the range of 
HEART RATE in their study - but on reflection it looks like we are putting words in the authors mouth so we have removed 'bursts of power required to maintain road position in traffic.' Which comes from our own experience and descriptions from the grey literature.

44) P14.L26-46. If the part regarding BMI was condensed substantially it would increase the focus of the manuscript.

Response: mostly removed now.

45) P15.L9: You write that you analyzed all participants together. What were the p-values for your unreported prior tests of interactions (gender and age)?

Response:? Not sure what to put here as a response as this section has been edited out - I guess that you would have liked us to provide $p$-values for differences between boys and girls? If this is deemed necessary we can do so but the means are available in table 1 , and hopefully the similarity (of z-scores at least) is a reassurance. None of the interactions were significant, but putting them in here is going to be a distraction I hope you'd agree?

46) P.15.L20: you write that you used a vertical jump test since normative values were available. How is this a logical argument? I can think of many other tests where normative values are available.

Response: true, it sounds pretty 'circular' now you point it out. What we meant was having, local (English), contemporary (2010) AND population-specific normative data for vertical jump and MEASRUES OF POWER (in addition to handgrip) enabled us to be consistent in our use of $z$ scores in our analyses. If we had used standing long jump data we would have had to use AVENA or HELENA study data from Spain or older US norms which would mess up our zscores and make them non-comparable between handgrip and jumps.

However, this was not the only reason for using a vertical jump as opposed to a long jump, it also allows the estimation of power, which the standing long jump does not - we have now added this argument. 


\section{Highlights.}

1. We examine the association between habitual school travel modality and children's muscular fitness

2. Walking to school is associated with greater jump peak power

3. Cycling to school is associated with greater handgrip strength

4. The likelihood of having good handgrip strength is independent of physical activity in cyclists

5. Active school transport may promote muscular fitness in youth. 


\section{Association between habitual school travel and muscular fitness in youth.}

Daniel Cohen ${ }^{1}$ Ayodele A Ogunleye ${ }^{2}$, Matthew Taylor ${ }^{2}$, Christine Voss ${ }^{3}$ Dominic

Micklewright $^{2} \&$ Gavin RH Sandercock ${ }^{2}$

\footnotetext{
${ }^{1}$ Instituto Masira, Faculty of Health Sciences, Medical School, University of Santander, Bucaramanga, Colombia

${ }^{2}$ School of Biological Sciences, University of Essex, Wivenhoe Park, Colchester CO3 3DU,

UK

${ }^{3}$ Centreer for Hip Health and Mobility, University of British Columbia, Vancouver, British Columbia, Canada.
}

Address for correspondence: Gavin RH Sandercock, Ph.D., School of Biological Sciences, University of Essex, Wivenhoe Park, Colchester, Essex, UK. CO4 3SQ; E-mail: gavins@essex.ac.uk. Tel: +44(0)1206-872043; Fax +44(0)1206-872592.

Running title: School travel and muscular fitness

$\begin{array}{ll}\text { Abstract word count: } & 186 \\ \text { Manuscript word count: } & 2895 \\ \text { Number of figures: } & 0 \\ \text { Number of tables: } & 3\end{array}$

This study was funded entirely by the University of Essex and no other sources of external funding were used. 


\begin{abstract}
.
Objective: To determine whether active school travel is associated with muscular fitness, which is an emerging marker of youth health. Methods: Handgrip strength, vertical jump and vertical jump peak power were measured in $n=6829$ English schoolchildren (53\% males, age $12.9 \pm 1.2$ years) between 2007 and 2011. Participants were grouped according to selfreported habitual school travel modality. Results: Cyclists had greater handgrip strength than passive travelers. Vertical jump height was greater in walkers and cyclists compared with passive travelers. Jump peak power was also higher in walkers than in the passive travel group. Compared with passive travelers, cyclists had a higher (age, sex and BMI-adjusted) likelihood of good handgrip strength (OR 1.42, 95\%CI;1.14-1.76) and walkers were more likely to have good measures for vertical jump peak power (OR 1.14, 95\%CI;1.00-1.29). Cyclists' likelihood of having good handgrip strength remained significantly higher when adjusted for physical activity (OR 1.29, 95\%CI;1.08-1.46). Conclusion: Muscular fitness differs according to school travel habits. Cycling is independently associated with better handgrip strength perhaps due to the physical demands of the activity. Better muscular fitness may provide another health-related reason to encourage active school travel.
\end{abstract}

Key words: HANDGRIP; PEAK POWER; CYCLING; ACTIVE TRAVEL; PHYSICAL ACTIVITY, ADOLESCENTS; CHILDREN 


\section{Highlights.}

1. We examine the association between habitual school travel modality and children's muscular fitness

2. Walking to school is associated with greater jump peak power

3. Cycling to school is associated with greater handgrip strength

4. The likelihood of having good handgrip strength is independent of physical activity in cyclists

5. Active school transport may promote muscular fitness in youth. 


\section{Introduction.}

The health-maintaining and protective the benefits of good cardiorespiratory fitness levels are established in adult (Sui et al., 2007, Blair et al., 2001) and pediatric populations (Ortega et al., 2008, Klasson-Heggebo et al., 2006, Hurtig-Wennlof et al., 2007, Steele et al., 2008). Recently, measures of strength such as isometric handgrip strength, and power such as jump performance, have been identified as powerful indicators of children's health status independent of cardiorespiratory fitness (Cohen et al., 2011, Benson et al., 2006, Artero et al., 2011, Moliner-Urdiales et al., 2011, Magnussen et al., 2012, Steene-Johannessen et al., 2009). Specifically, in children and adolescents with poor cardiorespiratory fitness, muscular strength is predictive of better insulin sensitivity (Benson et al., 2006) and lower prevalence and severity of clustered metabolic risk (Artero et al., 2011, Steene-Johannessen et al., 2009, Magnussen et al., 2012). Collective muscular fitness can be assessed using various performance tests of strength such as handgrip, explosive lower-limb power such as jumps, and muscular endurance such as sit-ups (Artero et al., 2011). Like cardiorespiratory fitness (Hunt et al., 2011, Blair et al., 1991, Pescatello et al., 2004), muscular fitness also appears to play a cardioprotective role, and is increasingly being recognized as an important health outcome, particularly in overweight and obese children (Artero et al., 2010, Artero et al., 2011, Cohen et al., 2014).

There is a robust relationship between handgrip strength in the lowest tertile (or quartile) of age-, sex- and body mass-adjusted performance (Artero et al., 2011, Ruiz et al., 2009). These tentative cut-points, described as healthy muscular fitness zones, are proposed because handgrip strength during adolescence is associated with current metabolic health (Artero et al., 2011) and future CVD risk and pre mature mortality (Ortega et al., 2012). Handgrip strength may act as a proxy of whole-body muscularity (Sherriff et al., 2009) and may be a useful addition to estimates of adiposity such as BMI. There is some evidence that, 
when used in isolation, BMI values are insensitive to changes in body composition and have previously reported that BMI remained stable over the 10-year period among children despite large, secular declines in muscular endurance (-30\% sit-up performance) and handgrip strength (-8\%) (Cohen et al., 2011). A systematical review reported global declines in jump power (Tomkinson, 2007), which is of concern given the emerging importance of muscular fitness as markers of current and future health.

As muscular fitness tracks from childhood into adulthood (McMillan \& Erdmann, 2010, Marshall et al., 1998) it is important to identify factors associated with its development in youth. Muscular fitness is related to adiposity (Deforche et al., 2003, Jimenez Pavon et al., 2010) and habitual physical activity (Moliner-Urdiales et al., 2010a) but these relationship are less widely reported than are the predictors of cardiorespiratory fitness.

School travel is one such example of a well-documented predictor of cardiorespiratory fitness in youth, and a recent systematic review of multiple international studies reported positive associations between active transport and cardiorespiratory fitness (Lubans et al., 2011). In contrast, only two studies have examined the association between school travel and elements of muscular fitness (Andersen et al., 2009, Ostergaard et al., 2013) but did report that children who travelled actively to school were stronger and had better muscular endurance than those who travelled by car or bus. These findings from Denmark, where cycling is the predominant modality for school travel are difficult to compare with countries like the US or UK, where cycling to school is rare. The aim of the present study was to determine if active school travel was associated with three measures of muscular fitness.

\section{Methods.}

\section{Participants}

The sampling strategy, population characteristics and methodology have been reported in 
detail previously (Voss \& Sandercock, 2010). The sample was drawn from the East of England Healthy Hearts Study and is restricted to individuals with complete, data for: sex, age (range 10.0-15.9 years), body mass index, cardiovascular end muscular fitness test performance, physical activity questionnaire and school travel habits ( $n=6989,53 \%$ males). The study was approved by the University of Essex Ethics Committee (Ref:FEC2007:0217), written parental consent and individual pupil's assent at point of testing. All participants were assessed during regularly scheduled physical education classes during the summer months of 2007-2011.

\section{Anthropometry}

We measured stature (Seca Leicester Height Measure; Seca GmbH \& Co. KG, Hamburg, Germany) to the nearest $1 \mathrm{~mm}$, and mass (Seca 888 digital scale; Seca GmbH \& Co. KG) to the nearest $0.1 \mathrm{~kg}$ with participants dressed in standard physical education clothing without shoes. We converted body mass index $\left(\mathrm{BMI}, \mathrm{kg} \cdot \mathrm{m}^{-2}\right)$ to age- and sex-specific $z$-scores (Cole et al., 1995).

\section{Muscular Fitness Measures}

After adjustment for hand-size, isometric handgrip strength of the dominant hand was measured using a portable dynamometer (Takei Corp Ltd., Tokyo, Japan) and converted to relative strength by dividing the highest score achieved by body mass $\left(\mathrm{W} \cdot \mathrm{kg}^{-1}\right)$. Handgrip strength was also expressed as a z-score using UK reference data (Cohen et al., 2010). A timing mat (NewTest Ltd. Oulu, Finland) measured flight time during a countermovement vertical jump (VJ) with the use of arms, which was expressed as VJ height (cm), and VJ peak power $\left(\mathrm{W} \cdot \mathrm{kg}^{-1}\right)$ based on the Sayers equation (Taylor et al., 2010). These measures were also converted to $z$-scores based on contemporary UK reference data (Taylor et al., 2010) . We created categorical variables of either 'poor or 'good' (handgrip strength, VJ height and 
peak power) according to whether z-scores fell in the lowest or upper two population tertiles respectively.

Cardiorespiratory Fitness.

We estimated peak oxygen consumption using the 20m shuttle-run test (20mSRT) administered as the FITNESSGRAM PACER, a modified version of the original protocol. Participants took part in the $20 \mathrm{mSRT}$ as part of their physical education. Cardiorespiratory fitness was expressed as estimated $\overline{\mathrm{VO}}_{2}$ peak $\left(\mathrm{ml} \cdot \mathrm{kg}^{-1} \cdot \mathrm{min}^{-1}\right)$ derived from the equation of Leger et al. (1988) from maximal running speed achieved (relative cardiorespiratory fitness) and also as z-scores (age-normalised cardiorespiratory fitness) based on UK reference data (Sandercock et al., 2012). A detail description of how $\dot{\mathrm{V}} \mathrm{O}_{2}$ peak was estimated available elsewhere (Sandercock et al., 2012).

\section{Measures of Physical Activity and School Travel}

We measured habitual physical activity using the Physical Activity Questionnaire for Adolescents (PAQ-A) or Children (PAQ-C) according to age. This 7-day recall instrument (Voss \& Sandercock, 2010) has acceptable criterion validity $\left(\mathrm{r}^{2}>.3\right.$; all components) (Kowalski et al., 2004) and does not assess school transport. Schoolchildren were also asked to self-report and to answer the question, "How do you usually get to school?", and given the following options: “walk," “cycle,", "public transport," “car'” or "'other.' The participants who selected "other' $(n=160)$ were excluded from the initial sample $(n=6829)$ grouped as: cycle, walk, public transport, or car.

We initially collapsed the responses into active (walk and cycle) $v s$. passive (car and public travel), then further divided the active travel group into those walked or cycled to school.

Participants also provided their home postcode which, together with their school postcode, we used Google Maps ${ }^{\mathrm{TM}}$ to calculate the modality-specific distance they travelled 
to school (e.g. use of footpaths for walkers, obeying local traffic laws for those driven). We dichotomized journey length as 'long' or 'short' based on median $(2.4 \mathrm{~km})$ split, in order to differentiate on the basis of measured distance rather travelling distance differences sometimes inferred from other methods such as primary-secondary school or age-related splits.

\section{Statistical Analyses}

We used Pearson's $\chi^{2}$ to assess differences in travel mode between sexes and independent samples t-tests to analyze between-sex differences in BMI, $\dot{\mathrm{V}} \mathrm{O}_{2}$ peak and muscular fitness tests (raw units and $z$-scores).

We used ANCOVA (controlling for: age, sex and BMI (z-score) with school (cluster) included as a random factor to quantify mean differences and effect sizes (Cohen's d) for comparison with existing research.

Finally we used binary logistic regression analysis to calculate odds ratios (OR) for the likelihood of being classed as having 'good' muscular fitness according to cycling or walking, using passive travel as the referent category. This multi-level analysis controlled for clustering at school level and for: age, sex and BMI (z-scores). A second model was created in which we also controlled for physical activity (PAQ-score). Statistical significance was set at $\mathrm{P}<0.05$. SPSS 18.0 for Windows (SPSS, Inc.: an IBM Company, Chicago, IL, USA) was used for all statistical analyses; except the multilevel logistic regression performed using the XTMELOGIC function of STATA 10.1 (StataCorp, TX, USA XTMELOGIT programme).

\section{Results.}

Table 1 shows the descriptive characteristics of the study population. BMI (z-scores) were elevated compared with reference data; and more-so in boys than girls. Absolute values for handgrip strength, VJ height and VJ peak power were higher in boys but age- and sex-normalized z-scores were more -comparable between sexes. 
Table 1. Participant descriptive characteristics and school travel habits.

\begin{tabular}{|c|c|c|c|c|c|c|}
\hline & \multicolumn{2}{|c|}{ All } & \multicolumn{2}{|c|}{ Boys } & \multicolumn{2}{|c|}{ Girls } \\
\hline & Mean or \% & SD or $n=$ & Mean or $\%$ & SD or $n=$ & Mean or \% & SD or $n=$ \\
\hline Number & 100 & (6829) & 53.2 & (3614) & 46.8 & (3215) \\
\hline Age (years) & 13.0 & $(1.4)$ & 13.1 & (1.5) & 13.0 & (1.5) \\
\hline $\mathrm{BMI}\left(\mathrm{kg} \cdot \mathrm{m}^{-2}\right)$ & 20.3 & (3.6) & 20.2 & (3.6) & 20.4 & (3.6) \\
\hline BMI (z-score) & 0.50 & $(1.1)$ & 0.58 & (1.1) & 0.51 & $(1.1)^{*}$ \\
\hline Handgrip strength (kg) & 24.4 & (7.9) & 26.1 & 9.0 & 21.9 & $(5.6)^{*}$ \\
\hline Handgrip strength (z-score) & -0.01 & $(1.0)$ & -0.02 & (1.0) & -0.01 & (1.0) \\
\hline VJ $(\mathrm{cm})$ & 28.0 & (7.7) & 30.4 & (7.7) & 26.0 & $(5.8)^{*}$ \\
\hline VJ (z-score) & -0.04 & $(1.1)$ & -0.03 & (1.1) & -0.06 & (1.1) \\
\hline VJ Peak Power $\left(\mathrm{W} \cdot \mathrm{kg}^{-1}\right)$ & 1818 & (729) & 1966 & (805) & 1644 & $(583)^{*}$ \\
\hline VJ Peak Power (z-score) & -0.16 & $(1.1)$ & -0.15 & (1.1) & -0.16 & (1.1) \\
\hline Physical Activity (PAQ-score) & 2.8 & $(0.7)$ & 3.0 & $(0.7)$ & 2.7 & $(0.6)^{*}$ \\
\hline Travel to school & 100 & (6829) & 53.2 & (3614) & 46.8 & (3215) \\
\hline Passive Transport & 44.9 & $(3062)$ & 44.3 & $(1602)$ & 45.6 & $(1460)$ \\
\hline Active Transport & 55.1 & $(3767)$ & 55.7 & (2012) & 54.4 & (1755) \\
\hline Of which: Walk & 47.1 & (3219) & 42.7 & $(1542)$ & 52.0 & $(1677)^{* *}$ \\
\hline Of which: Cycle & 8.0 & (348) & 13.0 & (269) & 2.5 & $(79)^{* *}$ \\
\hline
\end{tabular}

VJ; Vertical Jump, BMI z-score calculated UK1990 reference data (Cole et al., 1995). Handgrip strength z-scores based on UK reference data (Cohen et al., 2010). VJ and VJ peak power z-scores based UK reference data (Taylor et al., 2010). *Significant between-sex difference in means (t-test); ** Significant between-sex difference in frequencies $\left(\chi^{2}\right)$

Table 2. Means z-score $(95 \% \mathrm{Cl})$ for three indices of muscular fitness for walking, cycling or using passive school transport.

\begin{tabular}{|c|c|c|c|c|}
\hline Muscular Fitness Measure & Walk & Cycle & Passive & ANCOVA \\
\hline \multirow[t]{2}{*}{ Handgrip Strength $(\mathrm{z})^{\mathrm{a}}$} & -0.04 & $0.15^{*}$ & 0.01 & $\mathrm{~F}=10.1$, \\
\hline & $(-0.08$ to -0.01$)$ & (0.04 to 0.23$)$ & $(-0.04$ to 0.03$)$ & $P<0.001$ \\
\hline \multirow[t]{2}{*}{ VJ Height $(z)^{a}$} & $-0.01 *$ & $-0.02 *$ & -.11 & $F=4.4$ \\
\hline & $(-0.12 \text { to } 0.08)^{*}$ & $(-0.12$ to 0.08$)$ & $(-0.05$ to -0.14$)$ & $P=0.012$ \\
\hline \multirow[t]{2}{*}{ VJ Peak Power $(z)^{a}$} & $-0.14^{*}$ & -0.19 & -0.17 & $F=2.4$ \\
\hline & $(-0.18$ to -0.10$)$ & $(-0.29$ to 0.09$)$ & $(-0.22$ to -0.13$)$ & $P=0.07$ \\
\hline
\end{tabular}

VJ, Vertical Jump' a Controlling for age, sex and BMI (z-score); BMI z-score calculated UK1990 reference data (Cole et al., 1995) . Handgrip strength z-scores based on UK reference data (Cohen et al., 2010) . Vertical jump and jumping peak power z-scores based UK reference data (Taylor et al., 2010). *denotes significantly higher mean value than passive transport group. 
Table 3. Odds ratios $(95 \% \mathrm{Cl})$ of achieving good performance in three indices of muscular fitness.

\begin{tabular}{|c|c|c|c|}
\hline \multicolumn{4}{|c|}{ Model 1. (controlling for age, sex and BMI z-score) } \\
\hline & & OR $(95 \% \mathrm{Cl})$ & OR $(95 \% \mathrm{Cl})$ \\
\hline & Passive Transport & Walk & Cycle \\
\hline \multirow[t]{2}{*}{ Handgrip Strength } & Referent: 1 & 0.98 & 1.42 \\
\hline & & (0.88 to 1.10$)$ & (1.14 to 1.76$)$ \\
\hline \multirow[t]{2}{*}{ VJ Height } & Referent: 1 & 1.10 & 1.10 \\
\hline & & (0.97 to 1.26$)$ & (0.82 to 1.33$)$ \\
\hline \multirow[t]{2}{*}{ VJ Peak Power } & Referent: 1 & 1.14 & 1.04 \\
\hline & & (1.00 to 1.29$)$ & (0.83 to 1.32$)$ \\
\hline \multicolumn{4}{|c|}{ Model 2. (controlling for age, sex, BMI z-score and physical activity - PAQ) } \\
\hline & & OR (95\%CI) & OR (95\%CI) \\
\hline & Passive Transport & Walk & Cycle \\
\hline \multirow[t]{2}{*}{ Handgrip Strength } & Referent: 1 & 0.98 & 1.29 \\
\hline & & (0.87 to 1.08 ) & (1.08 to 1.46$)$ \\
\hline \multirow[t]{2}{*}{ VJ Height } & Referent: 1 & 0.92 & 1.11 \\
\hline & & (0.89 to 1.05 ) & (0.87 to 1.42 ) \\
\hline \multirow[t]{2}{*}{ VJ Peak Power } & Referent: 1 & 1.11 & 0.96 \\
\hline & & (0.98 to 1.26 ) & (0.76 to 1.21 ) \\
\hline
\end{tabular}

Multivariate analysis controlling for age, sex and BMI (z-score); VJ, Vertical Jump. BMI z-score calculated using UK 1990 reference data (Cole et al., 1995). Handgrip strength z-scores based on UK reference data (Cohen et al., 2010). Vertical jump height and vertical jump peak power z-scores based on UK reference data (Taylor et al., 2010). Low handgrip strength, vertical jump height and vertical jump peak power all classified as z-score within lowest tertile.

ANCOVA showed a significant main effect $\left(F_{(5,6824)}=10.1, p<0.001\right)$ for handgrip strength according to travel group (table 2). Post hoc tests of marginal means (Bonferroni for multiple comparison) showed significantly greater handgrip strength in than in the passive transport group.There was a main effect for $\mathrm{VJ}$ height $\left(F_{(5,6824)}=4.4, p=0.012\right)$ with significantly lower handgrip in the passive travel group compared with walkers and cyclists. As the main effect for VJ peak power was approaching statistical significance, we performed post hoc analysis and found that $\mathrm{VJ}$ peak power was significantly higher in walkers than in the passive travel group.

Mulitlevel binary logistic regression (age, sex and BMI (z-score)-adjusted) are shown in table 3. Cyclists were more likely ( $\mathrm{OR}=1.42,95 \% \mathrm{CI} ; 1.14$ to 1.76$)$ to have good handgrip strength compared with the passive travel group. These odds were attenuated but remained significant after controlling for physical activity (Model 2, OR=1.29, 95\%CI; 1.08 to 1.46). Walkers were more likely to have good VJ peak power ( $\mathrm{OR}=1.14,95 \% \mathrm{CI} ; 1.00$ to 1.29$)$ than 
passive travellers but controlling for physical activity (Model 2) attenuated these odds $(\mathrm{OR}=1.11,95 \% \mathrm{CI} ; 0.98$ to 1.26$)$.

\section{Discussion.}

The aim of this study was to determine if active school transport was associated with handgrip strength and VJ peak power, measures of muscular fitness related to health. The most comparable study (Andersen et al., 2009) to ours reported greater isometric muscle endurance (16\%) and abdominal muscle endurance (10\%) in Danish cyclists compared with passive commuters and a more recent analysis grouped by age and sex also reported greater isometric (back extensor) strength in active commuters (Ostergaard et al. 2013). We differences equivalent to $1 \mathrm{~kg}$ higher handgrip strength in cyclists which is likely to be clinically significant. Handgrip tracks from childhood to adulthood (Trudeau et al., 2003), and a recent meta-analysis (Cooper et al., 2010) suggested each $1 \mathrm{~kg}$ increment in age- and sex-adjusted handgrip strength lowers premature mortality risk by $3 \%$ (OR $0.97 ; 95 \% \mathrm{CI}$ 0.96-0.98).

We also found differences equivalent to a $1 \mathrm{~cm}$ greater $\mathrm{VJ}$ height, equating to a $35 \mathrm{~W}$ higher VJ peak power in walkers. All the between-group effect sizes are smaller $($ all $d<0.2)$ than reported for differences in cardiorespiratory fitness reported according to school travel (Voss \& Sandercock, 2010, Lubans et al., 2011, Andersen et al., 2009). An association with active travel and cardiorespiratory fitness seems logical as commuter cycling is a predominantly aerobic activity (Hendriksen et al., 2000). In adults, a period of commuter cycling led to a significantly increase in peak power/kg measured on a cycle ergometer (de Geus et al., 2009). We previously postulated a direct training effect from cycling to school on girls' cardiorespiratory fitness because the likelihood of female cyclists being fit was independent of their habitual physical activity (Voss \& Sandercock, 2010). Such training 
effects might be limited to the muscular activation pattern of the lower extremity during cycling and not be expressed in a vertical jump test which may explain the similarity in values between travel groups

The greater higher likelihood of good VJ peak power in those who walked to school was attenuated by controlling for physical activity (Model 2); a pattern reported previously for (boys) cardiorespiratory fitness (Voss \& Sandercock, 2010). Better lower body muscular fitness in walkers may be mediated by higher physical activity levels of active commuters as previously reported (Cooper et al., 2003).

The association between handgrip strength and cycling was independent of physical activity at other times possibly due to the neuromuscular demands of commuter cycling. Regular gripping and movement of handlebars and using mechanical brake levers may develop isometric strength in muscle-groups used in handgrip dynamometry. Cycling may also produce activation of the upper body musculature sufficient to stimulate the development of strength. Handgrip strength correlates well with maximum dynamic strength of upper and lower body muscle groups of adolescents (Milliken et al., 2008). Future research may wish to determine whether cyclists' greater handgrip strength actually indicates greater upper body strength or just more localized differences.

\section{Limitations and recommendations.}

Our cross-sectional design precludes inferences regarding cause and effect and there may be challenges to the validity of self-reported physical activity in younger participants. Despite the sample size, the number of (particularly female) cyclists remains low. Given the exploratory nature of our investigation we maximized statistical power by analyzing all participants using age- and sex-specific z-scores and further statistical adjustment. This approach is limited, not accounting for age-related differences in travel habits. While future 
studies would benefit from analyses stratified by sex and age they would require very large samples or deliberate oversampling of cyclists to ensure statistical power.

We used the vertical jump as our measure of lower body strength since as normative data were available and to estimate peak power by combining performance. Standing long jump may present a preferable alternative measure of lower limb power to vertical jumping as it correlates with metabolic health in youth (Garcia-Artero et al., 2007, Artero et al., 2011). Standing long jump, is more reliant on technique and can be difficult to interpret in youth as greater limb length is associated with better performance but greater body mass is associated with lower scores.

\section{Conclusions.}

These are the first data to show a greater likelihood of good muscular fitness in English children who commute actively to school. The association active commuting has with muscular fitness is weaker than that reported for cardiorespiratory fitness, but presents yet another potential reason to promote active school transport.

\section{Conflict of Interests: None}

\section{Acknowledgements}

The authors would like to thank the participating schools. This study was funded entirely by the University of Essex and no other sources of external funding were used. 


\section{References.}

Andersen, L. B., Lawlor, D. A., Cooper, A. R., Froberg, K. \& Anderssen, S. A. 2009. Physical fitness in relation to transport to school in adolescents: the Danish youth and sports study. Scand $J$ Med Sci Sports, 19, 406-11. doi: 10.1111/j.1600-0838.2008.00803.x.

Artero, E. G., Espana-Romero, V., Ortega, F. B., Jimenez-Pavon, D., Ruiz, J. R., Vicente-Rodriguez, G., Bueno, M., Marcos, A., Gomez-Martinez, S., Urzanqui, A., Gonzalez-Gross, M., Moreno, L. A., Gutierrez, A. \& Castillo, M. J. 2010. Health-related fitness in adolescents: underweight, and not only overweight, as an influencing factor. The AVENA study. Scand J Med Sci Sports, 20, 418-27. doi: 10.1111/j.1600-0838.2009.00959.x.

Artero, E. G., Ruiz, J. R., Ortega, F. B., Espana-Romero, V., Vicente-Rodriguez, G., Molnar, D., Gottrand, F., Gonzalez-Gross, M., Breidenassel, C., Moreno, L. A. \& Gutierrez, A. 2011. Muscular and cardiorespiratory fitness are independently associated with metabolic risk in adolescents: the HELENA study. Pediatr Diabetes. doi: 10.1111/j.1399-5448.2011.00769.x.

Benson, A. C., Torode, M. E. \& Singh, M. A. 2006. Muscular strength and cardiorespiratory fitness is associated with higher insulin sensitivity in children and adolescents. Int J Pediatr Obes, 1, 222-31

Blair, S. N., Cheng, Y. \& Holder, J. S. 2001. Is physical activity or physical fitness more important in defining health benefits? Med Sci Sports Exerc, 33, S379-99; discussion S419-20

Blair, S. N., Kohl, H. W., 3rd, Barlow, C. E. \& Gibbons, L. W. 1991. Physical fitness and all-cause mortality in hypertensive men. Ann Med, 23, 307-12

Castro-Piñero, J., Ortega, F. B., Artero, E. G., Girela-Rejón, M. J., Mora, J., Sjöström, M. \& Ruiz, J. R. 2010. Assessing Muscular Strength in Youth: Usefulness of Standing Long Jump as a General Index of Muscular Fitness. The Journal of Strength \& Conditioning Research, 24, 1810-1817 10.1519/JSC.0b013e3181ddb03d

Cohen, D., Voss, C., Taylor, M., Delextrat, A., Ogunleye, A. \& Sandercock, G. 2011. Ten-year secular changes in muscular fitness in English children. Acta Paediatr. doi: 10.1111/j.16512227.2011.02318.x.

Cohen, D. D., Gomez-Arbelaez, D., Camacho, P. A., Pinzon, S., Hormiga, C., Trejos-Suarez, J., Duperly, J. \& Lopez-Jaramillo, P. 2014. Low Muscle Strength Is Associated with Metabolic Risk Factors in Colombian Children: The ACFIES Study. Plos One, 9, e93150. doi: 10.1371/journal.pone.0093150PONE-D-13-48948 [pii].

Cohen, D. D., Voss, C., Taylor, M. J. D., Stasinopoulos, D. M., Delextrat, A. \& Sandercock, G. R. H. 2010. Handgrip strength in English schoolchildren. Acta Paediatrica, 99, 1065-1072. doi: DOI 10.1111/j.1651-2227.2010.01723.x.

Cole, T. J., Freeman, J. V. \& Preece, M. A. 1995. Body mass index reference curves for the UK, 1990. Arch Dis Child, 73, 25-9

Cooper, A., Wedderkopp, N., Jago, R., Kristensen, P., Moller, N., Froberg, K., Page, A. \& Andersen, L. 2008. Longitudinal associations of cycling to school with adolescent fitness. Prev Med, 324-328. doi: .

Cooper, A. R., Page, A. S., Foster, L. J. \& Qahwaji, D. 2003. Commuting to school: are children who walk more physically active? Am J Prev Med, 25, 273-6

Cooper, R., Kuh, D., Hardy, R., Mortality Review, G., Falcon \& Teams, H. A. S. 2010. Objectively measured physical capability levels and mortality: systematic review and meta-analysis. $B M J$, 341, c4467. doi: 10.1136/bmj.c4467.

de Geus, B., Joncheere, J. \& Meeusen, R. 2009. Commuter cycling: effect on physical performance in untrained men and women in Flanders: minimum dose to improve indexes of fitness. Scand $\boldsymbol{J}$ Med Sci Sports, 19, 179-87. doi: 10.1111/j.1600-0838.2008.00776.x.

Deforche, B., Lefevre, J., De Bourdeaudhuij, I., Hills, A. P., Duquet, W. \& Bouckaert, J. 2003. Physical fitness and physical activity in obese and nonobese Flemish youth. Obes Res, 11, 434-41

Garcia-Artero, E., Ortega, F. B., Ruiz, J. R., Mesa, J. L., Delgado, M., Gonzalez-Gross, M., GarciaFuentes, M., Vicente-Rodriguez, G., Gutierrez, A. \& Castillo, M. J. 2007. Lipid and 
metabolic profiles in adolescents are affected more by physical fitness than physical activity (AVENA study). Rev Esp Cardiol, 60, 581-8

Hendriksen, I. J., Zuiderveld, B., Kemper, H. C. \& Bezemer, P. D. 2000. Effect of commuter cycling on physical performance of male and female employees. Med Sci Sports Exerc, 32, 504-10

Hunt, L. P., Shield, J. P., Cooper, A. R., Ness, A. R. \& Lawlor, D. A. 2011. Blood pressure in children in relation to relative body fat composition and cardio-respiratory fitness. Int J Pediatr Obes, 6, 275-84. doi: 10.3109/17477166.2011.583655.

Hurtig-Wennlof, A., Ruiz, J. R., Harro, M. \& Sjostrom, M. 2007. Cardiorespiratory fitness relates more strongly than physical activity to cardiovascular disease risk factors in healthy children and adolescents: the European Youth Heart Study. Eur J Cardiovasc Prev Rehabil, 14, 57581. doi: 10.1097/HJR.0b013e32808c67e3.

Jimenez Pavon, D., Ortega, F. P., Ruiz, J. R., Espana Romero, V., Garcia Artero, E., Moliner Urdiales, D., Gomez Martinez, S., Vicente Rodriguez, G., Manios, Y., Beghin, L., Repasy, J., Sjostrom, M., Moreno, L. A., Gonzalez Gross, M. \& Castillo, M. J. 2010. Socioeconomic status influences physical fitness in European adolescents independently of body fat and physical activity: the HELENA study. Nutr Hosp, 25, 311-6. doi: S0212-16112010000200017 [pii].

Klasson-Heggebo, L., Andersen, L. B., Wennlof, A. H., Sardinha, L. B., Harro, M., Froberg, K. \& Anderssen, S. A. 2006. Graded associations between cardiorespiratory fitness, fatness, and blood pressure in children and adolescents. Br J Sports Med, 40, 25-9; discussion 25-9

Kowalski, K. C., Crocker, R. E. \& Donen, R. M. 2004. The Physical Activity Questionnaire for Older Children (PAC-C) and Adolescents (PAQ-A) Manual, Saskatoon, Canada, Universtiy of Saskatchewan.

Lubans, D. R., Boreham, C. A., Kelly, P. \& Foster, C. E. 2011. The relationship between active travel to school and health-related fitness in children and adolescents: a systematic review. Int $J$ Behav Nutr Phys Act, 8, 5. doi: 10.1186/1479-5868-8-5.

Magnussen, C. G., Schmidt, M. D., Dwyer, T. \& Venn, A. 2012. Muscular fitness and clustered cardiovascular disease risk in Australian youth. Eur J Appl Physiol, 112, 3167-71. doi: 10.1007/s00421-011-2286-4.

Marshall, S. J., Sarkin, J. A., Sallis, J. F. \& McKenzie, T. L. 1998. Tracking of health-related fitness components in youth ages 9 to 12. Medicine and Science in Sports and Exercise, 30, 910-916

Martinez-Gomez, D., Welk, G. J., Puertollano, M. A., Del-Campo, J., Moya, J. M., Marcos, A., Veiga, O. L. \& Group, A. S. 2011. Associations of physical activity with muscular fitness in adolescents. Scand J Med Sci Sports, 21, 310-7. doi: 10.1111/j.1600-0838.2009.01036.x.

McMillan, C. S. \& Erdmann, L. D. 2010. Tracking Adiposity and Health-Related Physical Fitness Test Performances From Early Childhood Through Elementary School. Pediatric Exercise Science, 22, 231-244

Milliken, L. A., Faigenbaum, A. D., Loud, R. L. \& Westcott, W. L. 2008. Correlates of upper and lower body muscular strength in children. J Strength Cond Res, 22, 1339-46. doi: 10.1519/JSC.0b013e31817393b1.

Moliner-Urdiales, D., Ortega, F. B., Vicente-Rodriguez, G., Rey-Lopez, J. P., Gracia-Marco, L., Widhalm, K., Sjostrom, M., Moreno, L. A., Castillo, M. J. \& Ruiz, J. R. 2010a. Association of physical activity with muscular strength and fat-free mass in adolescents: the HELENA study. Eur J Appl Physiol, 109, 1119-27. doi: 10.1007/s00421-010-1457-z.

Moliner-Urdiales, D., Ruiz, J. R., Ortega, F. B., Jimenez-Pavon, D., Vicente-Rodriguez, G., ReyLopez, J. P., Martinez-Gomez, D., Casajus, J. A., Mesana, M. I., Marcos, A., Noriega-Borge, M. J., Sjostrom, M., Castillo, M. J. \& Moreno, L. A. 2010b. Secular trends in health-related physical fitness in Spanish adolescents: the AVENA and HELENA studies. $J$ Sci Med Sport, 13, 584-8. doi: 10.1016/j.jsams.2010.03.004.

Moliner-Urdiales, D., Ruiz, J. R., Vicente-Rodriguez, G., Ortega, F. B., Rey-Lopez, J. P., EspanaRomero, V., Casajus, J. A., Molnar, D., Widhalm, K., Dallongeville, J., Gonzalez-Gross, M., Castillo, M. J., Sjostrom, M. \& Moreno, L. A. 2011. Associations of muscular and cardiorespiratory fitness with total and central body fat in adolescents: the HELENA study. Br J Sports Med, 45, 101-8. doi: 10.1136/bjsm.2009.062430. 
Ortega, F. B., Ruiz, J. R., Castillo, M. J. \& Sjostrom, M. 2008. Physical fitness in childhood and adolescence: a powerful marker of health. Int J Obes (Lond), 32, 1-11. doi: 10.1038/sj.ijo.0803774.

Ortega, F. B., Silventoinen, K., Tynelius, P. \& Rasmussen, F. 2012. Muscular strength in male adolescents and premature death: cohort study of one million participants. BMJ, 345, e7279. doi: 10.1136/bmj.e7279.

Ostergaard, L., Kolle, E., Steene-Johannessen, J., Anderssen, S. A. \& Andersen, L. B. 2013. Cross sectional analysis of the association between mode of school transportation and physical fitness in children and adolescents. Int J Behav Nutr Phys Act, 10, 91. doi: 10.1186/14795868-10-91.

Pescatello, L. S., Franklin, B. A., Fagard, R., Farquhar, W. B., Kelley, G. A. \& Ray, C. A. 2004. American College of Sports Medicine position stand. Exercise and hypertension. Med Sci Sports Exerc, 36, 533-53. doi: 00005768-200403000-00025 [pii].

Ruiz, J., Castro-Pinero, J., Artero, E. G., Ortega, F. B., Sjostrom, M., Suni, J. \& Castillo, M. J. 2009. Predictive validity of health-related fitness in youth: a systematic review. Br J Sports Med, 43, 909-23

Sandercock, G., Voss, C., Cohen, D., Taylor, M. \& Stasinopoulos, D. M. 2012. Centile curves and normative values for the twenty metre shuttle-run test in English schoolchildren. J Sports Sci, 30, 679-687. doi: 10.1080/02640414.2012.660185.

Sherriff, A., Wright, C. M., Reilly, J. J., McColl, J., Ness, A. \& Emmett, P. 2009. Age- and sexstandardised lean and fat indices derived from bioelectrical impedance analysis for ages 7-11 years: functional associations with cardio-respiratory fitness and grip strength. Br J Nutr, 101, 1753-60. doi: 10.1017/S0007114508135814.

Steele, R. M., Brage, S., Corder, K., Wareham, N. J. \& Ekelund, U. 2008. Physical activity, cardiorespiratory fitness, and the metabolic syndrome in youth. J Appl Physiol, 105, 342-51

Steene-Johannessen, J., Anderssen, S. A., Kolle, E. \& Andersen, L. B. 2009. Low muscle fitness is associated with metabolic risk in youth. Med Sci Sports Exerc, 41, 1361-7. doi: 10.1249/MSS.0b013e31819aaae5.

Sui, X., Laditka, J. N., Hardin, J. W. \& Blair, S. N. 2007. Estimated functional capacity predicts mortality in older adults. J Am Geriatr Soc, 55, 1940-7

Taylor, M. J., Cohen, D., Voss, C. \& Sandercock, G. R. 2010. Vertical jumping and leg power normative data for English school children aged 10-15 years. J Sports Sci, 28, 867-72. doi: 10.1080/02640411003770212.

Tomkinson, G. R. 2007. Global changes in anaerobic fitness test performance of children and adolescents (1958-2003). Scand J Med Sci Sports, 17, 497-507. doi: 10.1111/j.16000838.2006.00569.x.

Trudeau, F., Shephard, R. J., Arsenault, F. \& Laurencelle, L. 2003. Tracking of physical fitness from childhood to adulthood. Can J Appl Physiol, 28, 257-71

Voss, C. \& Sandercock, G. 2010. Aerobic fitness and mode of travel to school in English schoolchildren. Med Sci Sports Exerc, 42, 281-7. doi: 10.1249/MSS.0b013e3181b11bdc. 


\section{Preventive Medicine \\ Conflict of Interest Policy}

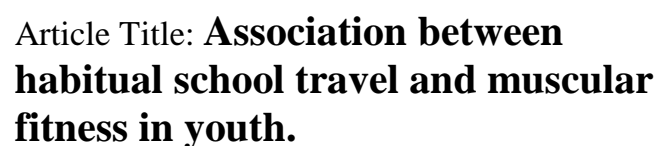

\section{Declarations}

Preventive Medicine requires that all authors sign a declaration of conflicting interests. If you have nothing to declare in any of these categories then this should be stated.

\section{Conflict of Interest}

A conflicting interest exists when professional judgement concerning a primary interest (such as patient's welfare or the validity of research) may be influenced by a secondary interest (such as financial gain or personal rivalry). It may arise for the authors when they have financial interest that may influence their interpretation of their results or those of others. Examples of potential conflicts of interest include employment, consultancies, stock ownership, honoraria, paid expert testimony, patent applications/registrations, and grants or other funding.

\section{Please state any competing interests}

None.

\section{Funding Source}

All sources of funding should also be acknowledged and you should declare any involvement of study sponsors in the study design; collection, analysis and interpretation of data; the writing of the manuscript; the decision to submit the manuscript for publication. If the study sponsors had no such involvement, this should be stated.

Please state any sources of funding for your research

None.

Signature (a scanned signature is acceptable,

Print name GAVIN SANDEROCK but each author must sign) 
ar $x \operatorname{han} \mathrm{h}$

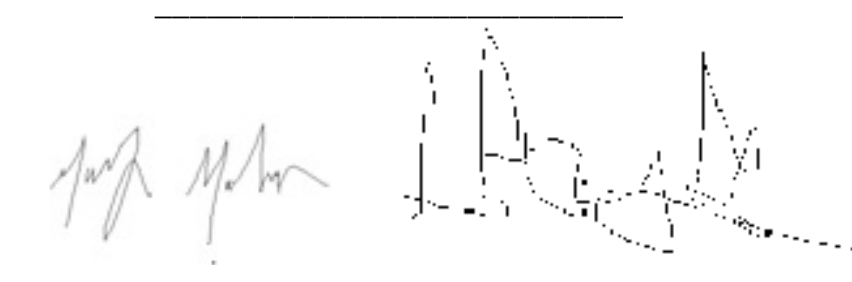

Waln abeo 